$1-1-2006$

\title{
Measurement of deeply virtual compton scattering with a polarized-proton target
}

S. Chen

Angela Biselli

Fairfield University, abiselli@fairfield.edu

CLAS Collaboration

Follow this and additional works at: https://digitalcommons.fairfield.edu/physics-facultypubs

Copyright American Physical Society Publisher final version available at http://prl.aps.org/ abstract/PRL/v97/i7/e072002

\section{Peer Reviewed}

\section{Repository Citation}

Chen, S.; Biselli, Angela; and CLAS Collaboration, "Measurement of deeply virtual compton scattering with a polarized-proton target" (2006). Physics Faculty Publications. 24.

https://digitalcommons.fairfield.edu/physics-facultypubs/24

\section{Published Citation}

Chen, S., et al. "Measurement of deeply virtual compton scattering with a polarized-proton target." Physical review letters 97.7 (2006): 072002. DOI: 10.1103/PhysRevLett.97.072002

This item has been accepted for inclusion in DigitalCommons@Fairfield by an authorized administrator of DigitalCommons@Fairfield. It is brought to you by DigitalCommons@Fairfield with permission from the rightsholder(s) and is protected by copyright and/or related rights. You are free to use this item in any way that is permitted by the copyright and related rights legislation that applies to your use. For other uses, you need to obtain permission from the rights-holder(s) directly, unless additional rights are indicated by a Creative Commons license in the record and/or on the work itself. For more information, please contact digitalcommons@fairfield.edu. 


\title{
Measurement of Deeply Virtual Compton Scattering with a Polarized-Proton Target
}

S. Chen, ${ }^{1}$ H. Avakian, ${ }^{2}$ V. D. Burkert, ${ }^{2}$ P. Eugenio, ${ }^{1}$ G. Adams,${ }^{31}$ M. Amarian, ${ }^{29}$ P. Ambrozewicz,${ }^{13}$ M. Anghinolfi, ${ }^{18}$ G. Asryan, ${ }^{39}$ H. Bagdasaryan, ${ }^{39,29}$ N. Baillie, ${ }^{38}$ J. P. Ball ${ }^{4}$ N. A. Baltzell, ${ }^{34}$ S. Barrow, ${ }^{1}$ V. Batourine,${ }^{22}$ M. Battaglieri, ${ }^{18}$ K. Beard, ${ }^{21}$ I. Bedlinskiy, ${ }^{20}$ M. Bektasoglu, ${ }^{29}$ M. Bellis,${ }^{31,7}$ N. Benmouna,,${ }^{14}$ B. L. Berman, ${ }^{14}$ A. S. Biselli, ${ }^{7}$ B. E. Bonner, ${ }^{32}$ S. Bouchigny, ${ }^{2,19}$ S. Boiarinov, ${ }^{20,2}$ P. Bosted, ${ }^{2}$ R. Bradford, ${ }^{7}$ D. Branford, ${ }^{12}$ W. J. Briscoe, ${ }^{14}$ W. K. Brooks, ${ }^{2}$ S. Bültmann, ${ }^{29}$ C. Butuceanu, ${ }^{38}$ J. R. Calarco, ${ }^{26}$ S. L. Careccia, ${ }^{29}$ D. S. Carman, ${ }^{28}$ B. Carnahan, ${ }^{8}$ A. Cazes,${ }^{34}$ P. L. Cole,${ }^{2,16}$ P. Collins, ${ }^{4}$ P. Coltharp, ${ }^{1}$ D. Cords,${ }^{2}$ P. Corvisiero, ${ }^{18}$ D. Crabb,${ }^{37}$ H. Crannell, ${ }^{8}$ V. Crede,${ }^{1}$ J. P. Cummings, ${ }^{31}$ R. De Masi, ${ }^{9}$ R. DeVita, ${ }^{18}$

E. De Sanctis, ${ }^{17}$ P. V. Degtyarenko, ${ }^{2}$ H. Denizli, ${ }^{30}$ L. Dennis, ${ }^{1}$ A. Deur, ${ }^{2}$ K. V. Dharmawardane, ${ }^{29}$ K. S. Dhuga, ${ }^{14}$ C. Djalali, ${ }^{34}$ G. E. Dodge,${ }^{29}$ J. Donnelly, ${ }^{15}$ D. Doughty, ${ }^{10,2}$ M. Dugger, ${ }^{4}$ S. Dytman, ${ }^{30}$ O. P. Dzyubak,${ }^{34}$ H. Egiyan, ${ }^{38,2}$ K. S. Egiyan ${ }^{39}$ L. El Fassi, ${ }^{3}$ L. Elouadrhiri, ${ }^{2}$ R. Fatemi, ${ }^{27}$ G. Fedotov, ${ }^{25}$ G. Feldman, ${ }^{14}$ R. J. Feuerbach, ${ }^{7}$ T. A. Forest, ${ }^{29}$ H. Funsten, ${ }^{38}$ M. Garçon, ${ }^{9}$ G. Gavalian, ${ }^{29}$ G. P. Gilfoyle, ${ }^{33}$ K. L. Giovanetti, ${ }^{21}$ F. X. Girod, ${ }^{9}$ J. T. Goetz, ${ }^{5}$ E. Golovatch, ${ }^{18}$ A. Gonenc, ${ }^{13}$ R. W. Gothe, ${ }^{34}$ K. A. Griffioen, ${ }^{38}$ M. Guidal, ${ }^{19}$ M. Guillo, ${ }^{34}$ N. Guler,${ }^{29}$ L. Guo, ${ }^{2}$ V. Gyurjyan, ${ }^{2}$

C. Hadjidakis, ${ }^{19}$ K. Hafidi, ${ }^{3}$ H. Hakobyan, ${ }^{39}$ R. S. Hakobyan, ${ }^{8}$ J. Hardie, ${ }^{10,2}$ D. Heddle, ${ }^{2}$ F. W. Hersman,${ }^{26}$ K. Hicks, ${ }^{28}$

I. Hleiqawi, ${ }^{28}$ M. Holtrop, ${ }^{26}$ M. Huertas, ${ }^{34}$ C. E. Hyde-Wright, ${ }^{29}$ Y. Ilieva, ${ }^{14}$ D. G. Ireland, ${ }^{15}$ B. S. Ishkhanov, ${ }^{25}$

E. L. Isupov, ${ }^{25}$ M. M. Ito, ${ }^{2}$ D. Jenkins, ${ }^{36}$ H. S. Jo, ${ }^{19}$ K. Joo, ${ }^{11}$ H. G. Juengst, ${ }^{29}$ C. Keith, ${ }^{2}$ J. D. Kellie, ${ }^{15}$ M. Khandaker,${ }^{27}$ K. Y. Kim, ${ }^{30}$ K. Kim, ${ }^{22}$ W. Kim, ${ }^{22}$ A. Klein, ${ }^{29}$ F. J. Klein, ${ }^{13,8}$ M. Klusman, ${ }^{31}$ M. Kossov,${ }^{20}$ L. H. Kramer, ${ }^{13,2}$

V. Kubarovsky, ${ }^{31}$ J. Kuhn, ${ }^{31,7}$ S. E. Kuhn, ${ }^{29}$ S. V. Kuleshov,${ }^{20}$ J. Lachniet, ${ }^{7,29}$ J. M. Laget, ${ }^{9,2}$ J. Langheinrich, ${ }^{34}$ D. Lawrence, ${ }^{24} \mathrm{Ji} \mathrm{Li},{ }^{31}$ A. C. S. Lima, ${ }^{14}$ K. Livingston, ${ }^{15} \mathrm{H}$. Lu, ${ }^{34}$ K. Lukashin, ${ }^{8}$ M. MacCormick,${ }^{19}$ N. Markov ${ }^{11}$ S. McAleer, ${ }^{1}$ B. McKinnon, ${ }^{15}$ J. W. C. McNabb, ${ }^{7}$ B. A. Mecking, ${ }^{2}$ M. D. Mestayer, ${ }^{2}$ C. A. Meyer, ${ }^{7}$ T. Mibe ${ }^{28}$ K. Mikhailov,${ }^{20}$ R. Minehart, ${ }^{37}$ M. Mirazita, ${ }^{17}$ R. Miskimen, ${ }^{24}$ V. Mokeev,${ }^{25}$ L. Morand, ${ }^{9}$ S. A. Morrow,,${ }^{19,9}$ M. Moteabbed, ${ }^{13}$ J. Mueller, ${ }^{30}$ G. S. Mutchler, ${ }^{32}$ P. Nadel-Turonski, ${ }^{14}$ J. Napolitano, ${ }^{31}$ R. Nasseripour, ${ }^{13,34}$ N. Natasha, ${ }^{39}$ S. Niccolai, ${ }^{14,19}$ G. Niculescu, ${ }^{28,21}$ I. Niculescu, ${ }^{14,21}$ B. B. Niczyporuk, ${ }^{2}$ M. R. Niroula, ${ }^{29}$ R. A. Niyazov, ${ }^{29,2}$ M. Nozar, ${ }^{2}$ G. V. O'Rielly, ${ }^{14}$ M. Osipenko, ${ }^{18,25}$ A. I. Ostrovidov, ${ }^{1}$ K. Park ${ }^{22}$ E. Pasyuk, ${ }^{4}$ C. Paterson, ${ }^{15}$ S. A. Philips, ${ }^{14}$ J. Pierce, ${ }^{37}$ N. Pivnyuk, ${ }^{20}$ D. Pocanic, ${ }^{37}$ O. Pogorelko, ${ }^{20}$ E. Polli, ${ }^{17}$ I. Popa,${ }^{14}$ S. Pozdniakov, ${ }^{20}$ B. M. Preedom, ${ }^{34}$ J. W. Price,,${ }^{5,6}$ Y. Prok ${ }^{37}$ D. Protopopescu ${ }^{26,15}$ L. M. Qin ${ }^{29}$ B. A. Raue, ${ }^{13,2}$ G. Riccardi, ${ }^{1}$ G. Ricco, ${ }^{18}$ M. Ripani, ${ }^{18}$ B. G. Ritchie, ${ }^{4}$ F. Ronchetti, ${ }^{17}$ G. Rosner, ${ }^{15}$ P. Rossi, ${ }^{17}$ D. Rowntree, ${ }^{23}$ P. D. Rubin,${ }^{33}$ F. Sabatié, ${ }^{29,9}$ C. Salgado, ${ }^{27}$ J. P. Santoro, ${ }^{2,36}$ V. Sapunenko, ${ }^{18,2}$ R. A. Schumacher, ${ }^{7}$ V. S. Serov, ${ }^{20}$ Y. G. Sharabian, ${ }^{2}$ J. Shaw, ${ }^{24}$ N. V. Shvedunov, ${ }^{25}$ A. V. Skabelin, ${ }^{23}$ E. S. Smith, ${ }^{2}$ L. C. Smith, ${ }^{37}$ D. I. Sober, ${ }^{8}$ A. Stavinsky, ${ }^{20}$ S. S. Stepanyan, ${ }^{22}$ S. Stepanyan, ${ }^{2}$ B. E. Stokes, ${ }^{1}$ P. Stoler, ${ }^{31}$ I. I. Strakovsky, ${ }^{14}$ S. Strauch, ${ }^{34}$ R. Suleiman, ${ }^{23}$ M. Taiuti, ${ }^{18}$ D. J. Tedeschi, ${ }^{34}$ U. Thoma, ${ }^{2}$ A. Tkabladze, ${ }^{14}$ S. Tkachenko, ${ }^{29}$ L. Todor, ${ }^{7}$ C. Tur, ${ }^{34}$ M. Ungaro, ${ }^{11}$ M. Vanderhaeghen,,${ }^{2,38}$ M. F. Vineyard, ${ }^{35,33}$ A. V. Vlassov,${ }^{20}$ D. P. Watts, ${ }^{15}$ L. B. Weinstein, ${ }^{29}$ D. P. Weygand, ${ }^{2}$ M. Williams, ${ }^{7}$ E. Wolin, ${ }^{2}$ M. H. Wood, ${ }^{34}$ A. Yegneswaran, ${ }^{2}$ J. Yun, ${ }^{29}$ L. Zana, ${ }^{26}$ J. Zhang, ${ }^{29}$ B. Zhao, ${ }^{11}$ and Z. Zhao ${ }^{34}$

\section{(CLAS Collaboration)}

\author{
${ }^{1}$ Florida State University, Tallahassee, Florida 32306, USA \\ ${ }^{2}$ Thomas Jefferson National Accelerator Facility, Newport News, Virginia 23606, USA \\ ${ }^{3}$ Argonne National Laboratory, Argonne, Illinois, 60439, USA \\ ${ }^{4}$ Arizona State University, Tempe, Arizona 85287-1504, USA \\ ${ }^{5}$ University of California at Los Angeles, Los Angeles, California 90095-1547, USA \\ ${ }^{6}$ California State University, Dominguez Hills, Carson, California 90747, USA \\ ${ }^{7}$ Carnegie Mellon University, Pittsburgh, Pennsylvania 15213, USA \\ ${ }^{8}$ Catholic University of America, Washington, DC 20064, USA \\ ${ }^{9}$ CEA-Saclay, Service de Physique Nucléaire, F91191 Gif-sur-Yvette, France \\ ${ }^{10}$ Christopher Newport University, Newport News, Virginia 23606, USA \\ ${ }^{11}$ University of Connecticut, Storrs, Connecticut 06269, USA \\ ${ }^{12}$ Edinburgh University, Edinburgh EH9 3JZ, United Kingdom \\ ${ }^{13}$ Florida International University, Miami, Florida 33199, USA \\ ${ }^{14}$ The George Washington University, Washington, DC 20052, USA \\ ${ }^{15}$ University of Glasgow, Glasgow G12 8QQ, United Kingdom \\ ${ }^{16}$ Idaho State University, Pocatello, Idaho 83209, USA \\ ${ }^{17}$ INFN, Laboratori Nazionali di Frascati, 00044 Frascati, Italy
}




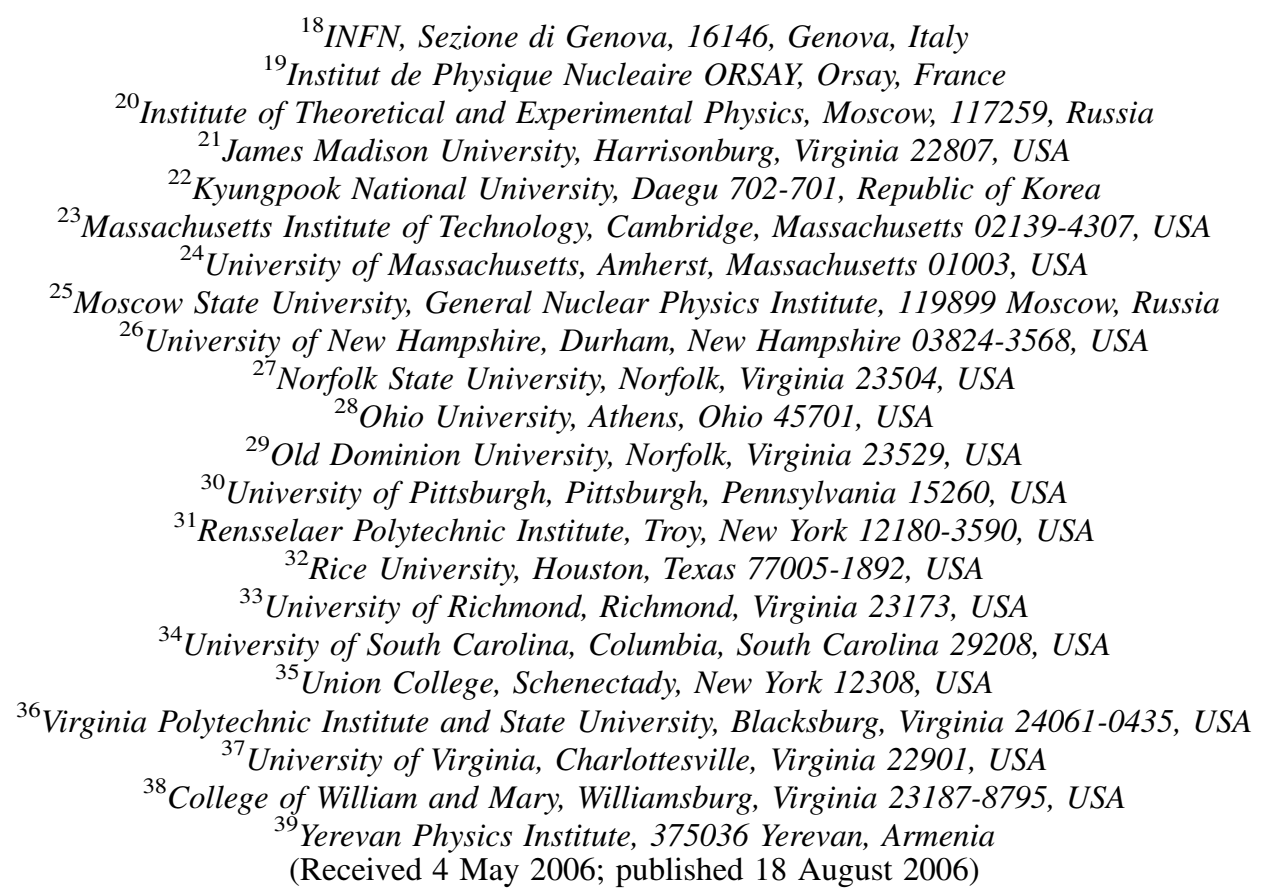

The longitudinal target-spin asymmetry $A_{\mathrm{UL}}$ for the exclusive electroproduction of high-energy photons was measured for the first time in $e \vec{p} \rightarrow e^{\prime} p \gamma$. The data have been accumulated at JLab with the CLAS spectrometer using $5.7 \mathrm{GeV}$ electrons and a longitudinally polarized $\mathrm{NH}_{3}$ target. A significant azimuthal angular dependence was observed, resulting from the interference of the deeply virtual Compton scattering and Bethe-Heitler processes. The amplitude of the $\sin \phi$ moment is $0.252 \pm 0.042^{\text {stat }} \pm$ $0.020^{\text {sys }}$. Theoretical calculations are in good agreement with the magnitude and the kinematic dependence of the target-spin asymmetry, which is sensitive to the generalized parton distributions $\tilde{H}$ and $H$.

Generalized parton distributions (GPDs) have in recent years been recognized as a versatile tool to investigate and describe the structure of hadrons at the quark-gluon level. They are closely related to conventional parton distributions and also to hadronic form factors, and contain information that cannot be accessed by either of these quantities. Important aspects where GPDs can provide new insight are the spatial distributions of quarks and gluons within the nucleon and the contribution of quark orbital angular momentum to the nucleon spin. GPDs contain the information needed to construct a multidimensional image of the internal structure of the nucleon. The role of GPDs in hard exclusive reactions and their relation to the nucleon's spatial structure and orbital angular momentum are discussed in detail in Refs. [1-8].

At high photon virtuality $Q^{2}$ and high-energy transfer $\nu$ (Bjorken scaling regime), the scattering amplitude for exclusive processes can be factorized into a hard scattering part (exactly calculable in perturbative QCD), and a nucleon structure part parametrized via GPDs. This process, called the "handbag approximation," is depicted in Fig. 1(a) for the case of high-energy photon production. In addition to the dependence on the parton momentum fraction $x$, GPDs depend on two more parameters, the fractional longitudinal momentum transfer $\xi$ to the quark [9], and the momentum transfer $t$ to the baryonic system.
One of the cleanest processes to access GPDs is deeply virtual Compton scattering (DVCS) in which one quark of the nucleon absorbs a virtual photon producing a real photon with the nucleon left intact. DVCS is most suitable for studying GPDs at moderate energies and in the valence quark regime. At low beam energies, the cross section for DVCS is small and masked by the more copious production of photons from the Bethe-Heitler $(\mathrm{BH})$ process. However, DVCS contributions can be measured directly through the interference of DVCS and BH amplitudes, which result in helicity-dependent cross section differences or asymmetries. The beam spin asymmetry and the targetspin asymmetry can be measured using a polarized elec-

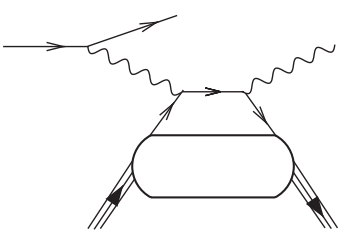

(a)

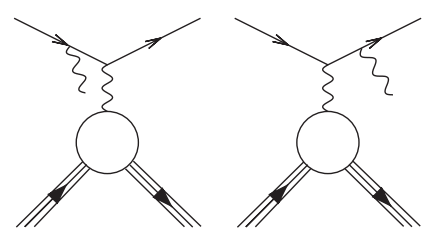

(b)
FIG. 1. Feynman diagrams for DVCS (a) and Bethe-Heitler processes (b) contributing to the amplitude of $e p \rightarrow e p \gamma$ scattering. 
tron beam or a polarized target. The two asymmetries are sensitive to different combinations of GPDs and thus provide complementary information [10]. First experimental results on the beam spin asymmetry $A_{\mathrm{LU}}$ with longitudinally polarized beam $(L)$ and unpolarized target $(U)$ resulting from the DVCS-BH interference have been reported by both the CLAS [11] and HERMES [12] collaborations.

In this Letter we present the first measurement of exclusive DVCS in the target-spin asymmetry measured in the reaction $e \vec{p} \rightarrow e^{\prime} p \gamma$. The target-spin asymmetry for unpolarized beam and longitudinally polarized target is defined as

$$
A_{\mathrm{UL}}(\phi)=\frac{d \sigma^{\Uparrow}(\phi)-d \sigma^{\Downarrow}(\phi)}{d \sigma^{\Uparrow}(\phi)+d \sigma^{\Downarrow}(\phi)},
$$

where $\Uparrow(\Downarrow)$ represents the target polarization antiparallel (parallel) to the beam direction, and $\phi$ is the angle between the electron scattering plane and the production plane [13]. The experiment measures the DVCS contribution through its interference with the Bethe-Heitler $(\mathrm{BH})$ process. In contrast to DVCS, where the photon is emitted from the nucleon, BH photons are emitted from the incoming or scattered electron (Fig. 1). While the BH cross section in most of the kinematic region is much larger than the DVCS cross section, the interference of the two contributions enhances the effect of DVCS and produces large cross section asymmetries for the target helicity aligned parallel or antiparallel with the electron beam. In the cross section difference, the helicity-independent $\mathrm{BH}$ contribution drops out and only the helicity-dependent interference term remains.

The asymmetry $A_{\mathrm{UL}}$ in leading order can be expressed in terms of GPDs [10]:

$$
\begin{aligned}
A_{\mathrm{UL}}(\phi) \propto & \left\{\xi\left(F_{1}+F_{2}\right)\left(\mathbf{H}+\frac{\xi}{1+\xi} \mathbf{E}\right)+F_{1} \tilde{\mathbf{H}}\right. \\
& \left.-\xi\left(\frac{\xi}{1+\xi} F_{1}+\frac{t}{4 M^{2}} F_{2}\right) \tilde{\mathbf{E}}\right\} \sin \phi,
\end{aligned}
$$

where $\tilde{\mathbf{H}}, \mathbf{H}, \tilde{\mathbf{E}}$, and $\mathbf{E}$ are sums over quark flavor of the corresponding GPDs with argument $x= \pm \xi$ [10], $F_{1}$ and $F_{2}$ are the known Dirac and Pauli form factors of the proton, and $M$ is the rest mass of the proton. In the range of this experiment the asymmetry is dominated by both $\mathbf{H}$ and $\tilde{\mathbf{H}}$, while $\tilde{\mathbf{E}}$ and $\mathbf{E}$ are kinematically suppressed. The effect of the $\phi$ dependence of the denominator on the value of the $\sin \phi$ moment extracted from the asymmetry was found negligible.

The data were taken from September 2000 to April 2001 using the CEBAF Large Acceptance Spectrometer (CLAS) [14]. CLAS is a multigap magnetic spectrometer equipped with drift chambers for track reconstruction, scintillator counters for time-of-flight measurements, electromagnetic calorimeters (EC) to identify electrons and photons, and Cherenkov counters (CC) for electron identification. The polar angle coverage of EC is from $8^{\circ}$ to $45^{\circ}$. Electrons at
$5.7 \mathrm{GeV}$ energy were incident on a longitudinally polarized ${ }^{15} \mathrm{NH}_{3}$ target. In this analysis, the asymmetry was averaged over the two beam helicities. The target [15] polarization was monitored online with a nuclear magnetic resonance (NMR) system, and ranged from $60 \%$ to $80 \%$. Unpolarized ${ }^{4} \mathrm{He}$ and ${ }^{12} \mathrm{C}$ targets were used to study the dilution due to the unpolarized material present in the polarized target.

The exclusive process $e p \gamma$ was determined by detecting all particles in the final state. Events were selected with the requirements that exactly one positive, one negative, and one neutral track were found for a given trigger, and the particle identifications for these tracks corresponded to an electron, a proton, and a photon, respectively. Deep inelastic kinematics was defined by selecting events with $Q^{2}>$ $1 \mathrm{GeV}^{2} / c^{2}, W>2 \mathrm{GeV} / c^{2}$, and $-t<0.6 \mathrm{GeV}^{2} / c^{2}$, where $W$ represents the photon-proton invariant mass.

For the ${ }^{15} \mathrm{NH}_{3}$ target, most of the events are from reactions on ${ }^{15} \mathrm{~N}$ (see Fig. 2). There is also a large background from $e \vec{p} \rightarrow e^{\prime} p \pi^{0}$ events where only one photon from the $\pi^{0}$ decay was detected. These backgrounds were suppressed by requiring that the detected photon in the overconstrained $e \vec{p} \rightarrow e^{\prime} p \gamma$ reaction was within 2 degrees of the photon angle predicted from the observed scattered $e^{\prime}$ and $p$ (see Fig. 2). The angle cut $\theta_{\gamma X}$ was defined based on Monte Carlo (MC) study. For further analysis events were selected within the missing mass $\vec{p}\left(e, e^{\prime} p\right) X$ range $-0.12\left(\mathrm{GeV} / c^{2}\right)^{2}<M_{X}^{2}<0.12\left(\mathrm{GeV} / c^{2}\right)^{2}$.

Figure 3(a) shows the azimuthal dependence of $A_{\mathrm{UL}}$, which is defined as

$$
A_{\mathrm{UL}}(\phi)=\frac{N^{\Uparrow}(\phi)-N^{\Downarrow}(\phi)}{f\left[P_{t}^{\Downarrow} N^{\Uparrow}(\phi)+P_{t}^{\Uparrow} N^{\Downarrow}(\phi)\right]},
$$

where $N^{\Uparrow}$ and $N^{\Downarrow}$ are the luminosity-normalized and acceptance-corrected numbers of $e \vec{p} \rightarrow e^{\prime} p \gamma$ events at

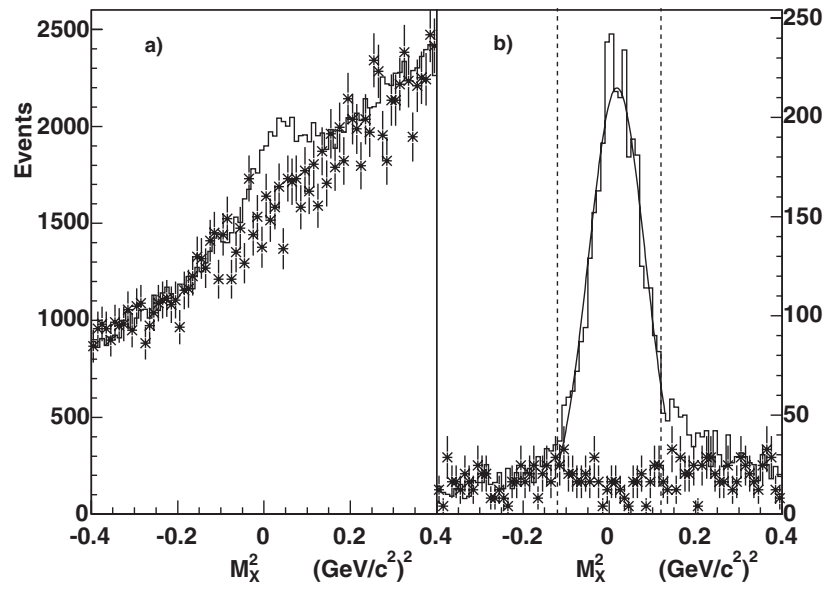

FIG. 2. Missing mass squared distribution of the system (ep) for reaction $e \vec{p} \rightarrow e^{\prime} p \gamma$. Panels (a) and (b) show the $M_{X}^{2}$ spectrum before and after the cut $\theta_{\gamma X}<2^{\circ}$. The ${ }^{12} \mathrm{C}$ data (stars) are normalized to the ${ }^{15} \mathrm{NH}_{3}$ data (solid line) using the negative tail of the $M_{X}^{2}$. The two dashed lines show the final cut on $M_{X}^{2}$ to select single photon production. 


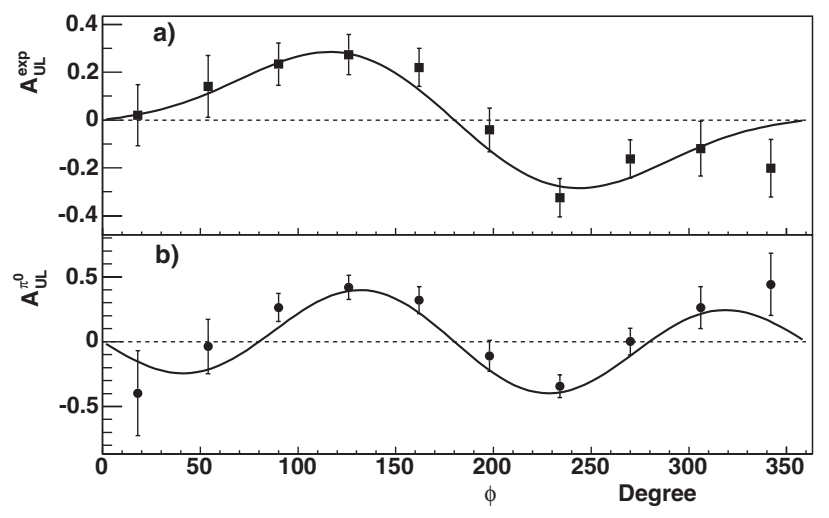

FIG. 3. Azimuthal angle $\phi$ dependence of the measured targetspin asymmetry for photons (a) and $\pi^{0}$ (b). The solid curves represent the fitted function $\alpha \sin \phi+\beta \sin 2 \phi$ with parameters $\alpha=0.240 \pm 0.042$ and $\beta=-0.087 \pm 0.045$ (a), and $\alpha=$ $0.109 \pm 0.056$ and $\beta=-0.319 \pm 0.061$ (b). In (a), the photon events are still contaminated by $\pi^{0}$ events.

positive and negative target helicity, respectively, $P_{t}^{\Uparrow}$ and $P_{t}^{\Downarrow}$ are absolute values of the corresponding target polarizations, and $f=0.901 \pm 0.035$ is the dilution factor, which is defined as the ratio of the number of events from hydrogen and from $\mathrm{NH}_{3}$.

The above photon event sample remains contaminated by photons from $\pi^{0}$ decays that were not removed by the angle cut. In order to correct for this contamination, we analyzed $\pi^{0}$ events in the same kinematic range as the single $\gamma$ events. Events were selected requiring one electron, one proton, and two detected photons. Most of these events are from nuclear protons, for which the squared missing mass $M_{X}^{2}$ is much different from the nominal $M_{\pi^{0}}^{2}=0.018\left(\mathrm{GeV} / c^{2}\right)^{2}$. Using a similar technique as was used in the DVCS-BH event selection, we placed a cut on the difference of the measured and the predicted $\pi^{0}$ angles of $\theta_{\pi^{0} X}<2.5^{\circ}$, where the $\pi^{0}$ angle was reconstructed from measured photons, while the angle of $X$ is predicted using 4-momentum conservation for $e \vec{p} \rightarrow e^{\prime} p X$ assuming free proton kinematics. The $\pi^{0}$ events were further selected with cuts $0.05 \mathrm{GeV} / c<M_{\gamma \gamma}<0.18 \mathrm{GeV} / c$ and $-0.1\left(\mathrm{GeV} / c^{2}\right)^{2}<M_{X}^{2}<0.14\left(\mathrm{GeV} / c^{2}\right)^{2}$. For the identified $\pi^{0}$ events, the dilution factor was $f=0.782 \pm 0.036$.

Figure 3(b) shows the azimuthal dependence of $A_{\mathrm{UL}}^{\pi^{0}}$, which was used to correct the DVCS asymmetry for $\pi^{0}$ contamination. We note that the asymmetry for $\pi^{0}$ production has a dominant $\sin 2 \phi$ dependence while the asymmetry for photon production has a dominant $\sin \phi$ dependence even before $\pi^{0}$ contributions are fully removed from the single photon sample. To estimate the remaining $\pi^{0}$ contamination in the single $\gamma$ events, a MC study was performed. DVCS-BH and $\pi^{0}$ events were generated and passed through GSIM, the GEANT-based simulation software package of the CLAS spectrometer. The output of GSIM was processed using the same procedure as was used for the data. The MC $\pi^{0}$ spectrum was normalized to the number of $\pi^{0}$ events observed in the data. Following the same procedure as was used in the selection of DVCS-BH events, $\pi^{0}$ events with only one photon detected were selected to simulate the background from $\pi^{0}$. The $\phi$ dependence of the $\pi^{0}$ fraction $\left(F_{\pi^{0}}\right)$ is shown in Table I.

Finally, the fully corrected target-spin asymmetry for single $\gamma$ production was determined using equation:

$$
A_{\mathrm{UL}}^{\mathrm{exp}}(\phi)=F_{\gamma}(\phi) A_{\mathrm{UL}}(\phi)+F_{\pi^{0}}(\phi) A_{\mathrm{UL}}^{\pi^{0}}(\phi),
$$

where $A_{\mathrm{UL}}^{\exp }$ is the experimentally measured asymmetry with the $\pi^{0}$ background as shown in Fig. 3(a), $A_{\mathrm{UL}}^{\pi^{0}}$ is the target-spin asymmetry for $\pi^{0}$ as shown in Fig. 3(b), and $F_{\gamma}=1-F_{\pi^{0}}$ is the fraction of DVCS-BH.

The azimuthal dependence of the final asymmetry $A_{\mathrm{UL}}$ is shown in Fig. 4 at $\left\langle Q^{2}\right\rangle=1.82 \mathrm{GeV}^{2} / c^{2},\langle-t\rangle=$ $0.31 \mathrm{GeV}^{2} / c^{2}$, and $\langle\xi\rangle=0.16$. The $\phi$ dependence was fitted with the function $\alpha \sin \phi+\beta \sin 2 \phi$ (solid curve) with parameters $\alpha=0.252 \pm 0.042^{\text {stat }} \pm 0.020^{\text {sys }}$, and $\beta=-0.022 \pm 0.045^{\text {stat }} \pm 0.021^{\text {sys }}$. The $A_{\mathrm{UL}}$ is dominated by the $\sin \phi$ term while the $\sin 2 \phi$ term is compatible with zero within the error bars, indicating that higher twists do not contribute significantly in our kinematical range.

To obtain information on the kinematic dependence of the $\sin \phi$ moment of $A_{\mathrm{UL}}\left(A_{\mathrm{UL}}^{\sin \phi}\right)$ [13], the data were divided into 3 bins in $\xi$ and $-t$, respectively. The leading term $A_{\mathrm{UL}}^{\mathrm{sin} \phi}$ was extracted for each bin. The results are shown in Fig. 5, where the asymmetry was integrated over the other kinematic variables. A clear $\xi$ dependence of $A_{\mathrm{UL}}^{\sin \phi}$ is seen, with asymmetries increasing with $\xi$. The theoretical calculations shown in Figs. 4 and 5 have been obtained by including target mass corrections. Unlike deep inelastic scattering (DIS), a full calculation of such corrections is still an open problem for DVCS. We have, however, included the kinematical higher twist effects in the twist-2 amplitude. In the presence of those effects the GPDs entering in the asymmetry Eq. (2) are proportional to GPDs at $\left(\xi^{\prime}, \xi, t\right)$, where the difference between $\xi^{\prime}$ and $\xi$ include terms proportional to $M^{2} / Q^{2}$ and $-t / Q^{2}$ as shown in Ref. [9]. As can be noticed on Figs. 4 and 5, the thus obtained theoretical calculation agrees within experimental uncertainties well with the measurement.

In Figs. 4 and 5, the error bars are statistical, and the systematic uncertainty is shown as a band at the bottom. The sources of systematic uncertainties are identified as the dilution factor calculation $(\sim 4 \%)$, estimation of target

TABLE I. The $\pi^{0}$ fraction and statistical uncertainties in observed single photon events.

\begin{tabular}{cccc}
\hline \hline$\phi$ (degree) & $F_{\pi^{0}} \pm \Delta F_{\pi^{0}}$ & $\phi$ (degree) & $F_{\pi^{0}} \pm \Delta F_{\pi^{0}}$ \\
\hline $0-36$ & $0.106 \pm 0.010$ & $180-216$ & $0.373 \pm 0.022$ \\
$36-72$ & $0.117 \pm 0.009$ & $216-252$ & $0.313 \pm 0.019$ \\
$72-108$ & $0.242 \pm 0.018$ & $252-288$ & $0.216 \pm 0.015$ \\
$108-144$ & $0.324 \pm 0.021$ & $288-324$ & $0.103 \pm 0.008$ \\
$144-180$ & $0.414 \pm 0.023$ & $324-360$ & $0.101 \pm 0.007$ \\
\hline \hline
\end{tabular}




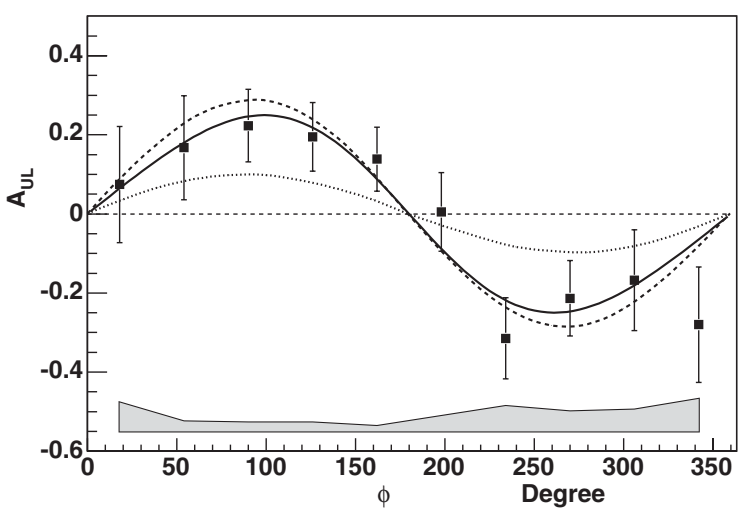

FIG. 4. The azimuthal angle $\phi$ dependence of the target-spin asymmetry for exclusive electroproduction of photons after subtraction of the $\pi^{0}$ background. The dashed curve is the full model prediction using the $\xi$-dependent GPD parametrization [9] ( $b_{\text {val }}=b_{\text {sea }}=1$, and $E=\tilde{E}=0$ ) based on MRST02 unpolarized PDFs [20] and polarized PDFs [21] for the twist-2 terms, and higher twists included in those terms. The dotted curve shows the asymmetry when $\tilde{H}=0$. The solid curve is described in the text.

polarization $(\sim 7 \%),{ }^{15} \mathrm{~N}$ polarization $(\sim 0.5 \%)$ [16], radiative corrections $(<0.1 \%)$ [17], evaluation of the $\pi^{0}$-decay background from $\mathrm{MC}$ simulations $(<2.5 \%)$, and the angle cut $(<5 \%)$.

Combined with the data expected from precision measurements of the beam spin asymmetry which is dominated by GPD $H$ [18], these results will allow us to constrain different GPDs. The target-spin asymmetry in DVCS is also under study at HERMES [19].

In summary, we have presented the target-spin asymmetry for exclusive electroproduction of photons. A significant $\sin \phi$ moment of the target-spin asymmetry is observed and is consistent with predictions based on the GPD formalism. The measured asymmetry is consistent with predictions of a large contribution from GPD $\tilde{H}$.

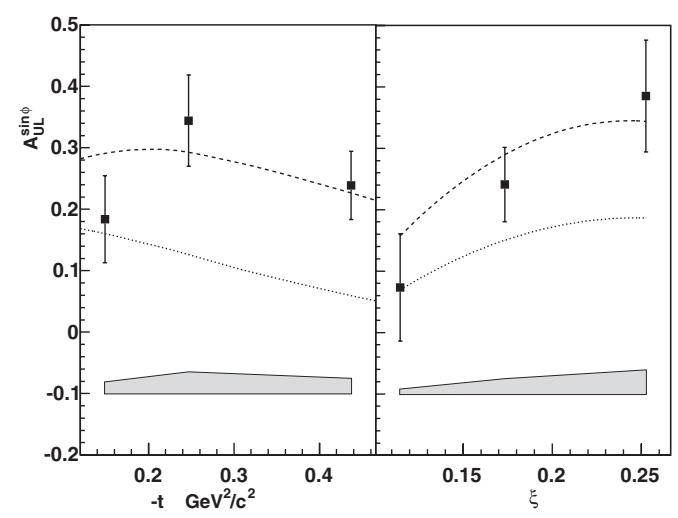

FIG. 5. The left panel shows the $-t$ dependence of the $\sin \phi$ moment of $A_{\mathrm{UL}}$ for exclusive electroproduction of photons, while the right shows the $\xi$ dependence. Curves as in Fig. 4.
Kinematic dependences of the target-spin asymmetry have also been studied. The leading term $A_{\mathrm{UL}}^{\sin \phi}$ increases with increasing $\xi$, in agreement with the model prediction.

We gratefully acknowledge the outstanding efforts of the staff of the Accelerator and Physics Division at Jefferson Lab that made this experiment possible. This work was supported in part by the U.S. Department of Energy (No. DE-FG02-92ER40735) and the National Science Foundation, the Italian Istituto Nazionale di Fisica Nucleare, the French Centre National de la Recherche Scientifique, the French Commissariat à l'Energie Atomique, the UK Engineering and Physical Science Research Council, and the Korean Research Foundation. The Southeastern Universities Research Association (SURA) operates the Thomas Jefferson National Accelerator Facility for the United States Department of Energy under Contract No. DE-AC05-84ER40150.

[1] D. Müller et al., Fortschr. Phys. 42, 101 (1994).

[2] X. Ji, Phys. Rev. Lett. 78, 610 (1997).

[3] A. V. Radyushkin, Phys. Lett. B 380, 417 (1996); Phys. Rev. D 56, 5524 (1997).

[4] K. Goeke, M. Polyakov, and M. Vanderhaeghen, Prog. Part. Nucl. Phys. 47, 401 (2001).

[5] M. Diehl, Phys. Rep. 388, 41 (2003).

[6] A. Belitsky, X. Ji, and F. Yuan, Phys. Rev. D 69, 074014 (2004).

[7] A. Belitsky and A. Radyushkin, Phys. Rep. 418, 1 (2005).

[8] M. Burkardt, Int. J. Mod. Phys. A 18, 173 (2003); 21, 926 (2006).

[9] M. Vanderhaeghen, P. A. M. Guichon, and M. Guidal, Phys. Rev. D 60, 094017 (1999).

[10] A. Belitsky, D. Müller, and A. Kirchner, Nucl. Phys. B629, 323 (2002).

[11] S. Stepanyan et al., Phys. Rev. Lett. 87, 182002 (2001).

[12] A. Airapetian et al., Phys. Rev. Lett. 87, 182001 (2001).

[13] A. Bacchetta et al., Phys. Rev. D 70, 117504 (2004).

[14] B. A. Mecking et al., Nucl. Instrum. Methods Phys. Res., Sect. A 503, 513 (2003).

[15] C. D. Keith et al., Nucl. Instrum. Methods Phys. Res., Sect. A 501, 327 (2003).

[16] D. G. Crabb et al., Nucl. Instrum. Methods Phys. Res., Sect. A 356, 9 (1995).

[17] A. V. Afanasev, M.I. Konchatnij, and N.P. Merenkov, J. Exp. Theor. Phys. 102, 220 (2006).

[18] CEBAF experiments E-01-113/06-003 (CLAS) and E-00110 (Hall A). More information on these experiments is available at: http://www.jlab.org/exp_prog/generated/apphalla.html, http://www.jlab.org/exp_prog/generated/apphallb.html.

[19] M. Kopytin (HERMES Collaboration), 13th International Workshop on Deep Inelastic Scattering, AIP Conference Proceedings Vol. 792 (AIP, New York, 2005).

[20] A. D. Martin, R. G. Roberts, W. J. Stirling, and R. S. Thorne, Phys. Lett. B 531, 216 (2002).

[21] E. Leader, A. V. Sidorov, and D. B. Stamenov, Phys. Rev. D 58, 114028 (1998). 